\title{
Das Austrian Law Journal - ein Erfahrungsbericht
}

\author{
Elisabeth Staudegger*
}

A. Einleitung ........................ 161

B. Gründung und Grundlagen des ALJ ... 161

I. Motivation: Qualitätssicherung in der rechtswissenschaftlichen For-

schung ............................ 161

II. Ziel ................................ 163

III. Organisatorische Umsetzung ........ 164

IV. Technische Umsetzung .............. 164

V. Zusammenarbeit mit der Universitätsbibliothek ....................... 165

VI. Finanzierung $\ldots \ldots \ldots \ldots \ldots \ldots \ldots \ldots \ldots \ldots$

C. Betrieb $2014-2018$................. 165

I. Struktur ........................... 166

II. Rechtsrahmen .................... 166

III. Organisatorische und institutionelle Verfestigung
IV. Publikationen 168

V. Organisation und nachhaltige Dokumentation von Veranstaltungen .............................. 169

VI. Nutzungsstatistik ................... 169

VIII. Stand September 2019 ............. 170

D. Best und Worst Practices sowie offene

Themen ............................. 171

I. Best und Worst Practices ............ 171

II. Anonymität des peer review Prozesses ............................. 173

III. Nutzung weiterer IT-Möglichkeiten ............................. 174

IV. Unentgeltlichkeit des Angebots / Open Science ........................ 174

E. Fazit ............................. 176

\section{A. Einleitung}

Die Einladung, im fünften Jahr des Bestehens einen Erfahrungsbericht zu Entstehung und Entwicklung des Austrian Law Journal (ALJ) beizutragen, nehmen wir gerne an. Der Beitrag geht einerseits auf die Motivation ein, die zur Gründung eines Open Access Journals im Bereich der österr Rechtswissenschaften geführt hat; andererseits werden die Herausforderungen, die sich bei der Realisierung und im Betrieb gezeigt haben, beschrieben. Ziel ist es dabei, die best, aber auch worst practices offenzulegen und damit anderen Initiativen sowohl als gutes Beispiel zu dienen, als auch die Vermeidung ähnlicher Fehler zu erleichtern.

\section{B. Gründung und Grundlagen des ALJ}

\section{Motivation: Qualitätssicherung in der rechtswissenschaftlichen Forschung}

Die Gründung des ALJ ging auf das seitens des Wissenschaftsministeriums geäuBerte Anliegen zurück, rechtswissenschaftliche Forschung nach objektiven Kriterien evaluieren zu wollen. Tatsächlich fehlten (und fehlen bis heute) in Österreich messbare, den aus den natur- und geisteswissenschaftlichen Disziplinen bekannten „Impact-Faktoren“ vergleichbare Qualitätskriterien in den österreichischen Rechtswissenschaften. ${ }^{1}$ Rechtswissenschaftliche Forschung wird gemeinhin viel-

* Elisabeth Staudegger ist Leiterin des Fachbereichs Recht und IT am Institut für Rechtswissenschaftliche Grundlagen der Universität Graz und Gründungsmitglied bzw aktuell CoEditor des ALJ.

1 Zwar behandelt die am Institut für öffentliches Recht und Politikwissenschaft der Universität Graz verfasste Dissertation von Maier, Bewertung juristischer Forschung, rechtliche Grundlagen und Praxis (2018) das Thema vertiefend. In der Praxis sind anerkannte Kriterien derzeit allerdings nicht auffindbar. 
mehr dann als erfolgreich bewertet, wenn die vertretenen Meinungen von den Höchstgerichten aufgegriffen und die ForscherInnen in den Entscheidungen zitiert werden. Dass damit weit überwiegend die die Rechtsanwendung unmittelbar unterstützende Dogmatik erfassbar ist, steht außer Zweifel. Ähnliches gilt für die Erwähnung in den Materialien im Zuge des Gesetzwerdungsprozesses. Selbstverständlich gibt es aber auch in Österreich rege Publikationstätigkeiten, insb in Zeitschriften, sodass eine Anlehnung an die naturwissenschaftliche Praxis durchaus überlegenswert schien.

Die im Jahr 2012 dazu an der rechtswissenschaftlichen Fakultät in Graz eingerichtete Arbeitsgruppe scheiterte jedoch am Versuch, ein von der Mehrheit der ForscherInnen getragenes „Ranking“ der mehr als 100 juristischen Fachzeitschriften ${ }^{2}$ in Österreich aufzustellen. Der damalige Forschungsdekan, Stefan Storr, lud daraufhin zu einem ganz grundlegenden Arbeitstreffen, in dem die Möglichkeiten sinnvoller Forschungsevaluierung in den Rechtswissenschaften diskutiert werden sollten. An diesem Arbeitstreffen beteiligten sich insb Brigitta Lurger und die Verfasserin dieses Beitrags - womit die Trias der Gründungsmitglieder des ALJ gefunden war.

Dabei wurden fünf Beobachtungen zu einem deutlich veränderten wissenschaftlichen Publikationswesen festgestellt: ${ }^{3}$

1. Die Ausrichtung juristischer Fachzeitschriften auf den Leserkreis der Rechtspraxis.

Eine juristische Fachzeitschrift, die in erster Linie auf Vermittlung wissenschaftlicher Erkenntnis und wissenschaftlicher Diskussion ausgerichtet ist, fehlt.

2. Qualität statt Quantität. Wir halten den Grundsatz „publish or perish“ für wissenschaftliche Karrieren für überkommen.

3. „Publikationsmärkte“ erwarten Entscheidungsbesprechungen und Gesetzgebungsmonitoring. Die Rechtswissenschaften sollten sich (jedenfalls auch) wieder stärker auf die eigentliche Forschung konzentrieren.

4. Der Europäisierung und Internationalisierung der Rechtswissenschaften wird im Hinblick auf die Dissemination der Forschungsergebnisse wenig Rechnung getragen.

5. Es gibt kaum Open Access-Zeitschriften in den österr Rechtswissenschaften.

2 Schon im Jahr 2014 erzeugten und vertrieben im Wesentlichen die Verlage Manz, LexisNexis, Linde und Verlag Österreich sowie wenige kleinere Verlage wie zB Jan Sramek und Medien\&Recht die österr juristischen Fachzeitschriften. Hinweise auf das aktuelle Verlagsangebot finden sich in den jeweiligen Verlagswebsites. Eine umfassende Übersicht über das gesamte Zeitschriftenangebot bietet die verlagsunabhängige Rechtsindexdatenbank RIDA unter <http://www.ridaonline.at> (15.9.2019).

3 Die Details zur Gründungsphase des ALJ basieren weitgehend auf dem Konzeptpapier „Austrian Law Journal“ 3.10.2013 idF 19.2.2014. 
Eine Analyse der Entwicklungen in den Geistes- und Naturwissenschaften zeigte jedoch deutlich, welche Macht Wissenschaftsverlagen eingeräumt wird, wenn die Karriere der NachwuchswissenschaftlerInnen an Impact-Faktoren gekoppelt wird. Auch führt das System zu wesentlichen Verzerrungen der Forschungsergebnisse. ${ }^{4}$ Gleichzeitig wiesen schon damals die Bibliotheken auf die zunehmende Teuerung hin, die manche Wissenschaftsprodukte beinahe unerschwinglich machten. Und schließlich werden ernste Zweifel an der Tauglichkeit der Impact-Faktor geleiteten Messmethoden laut. Hingegen wurde das als „double blind peer review“ bekannte Verfahren der wissenschaftsinternen gutachterlichen Stellungnahme zu geplanten Veröffentlichungen als wertvolle Bereicherung des Publikationsprozesses aufgegriffen.

Dieser Ausgangslage Rechnung tragend, sollte mit dem „Austrian Law Journal Open Access Review of Developments in Austrian, European and International Law" ein Publikationsmedium gegründet werden, das explizit eine neue Veröffentlichungskultur in den Rechtswissenschaften fördert.

\section{Ziel}

Die Idee, anstelle des Gewichtens bestehender Verlagsprodukte eine eigene Plattform für die Publikation von Forschungsergebnissen zu schaffen und dabei das anonymisierte Gutachtensverfahren einzusetzen, fand breiten Anklang.

Grundlegend war, dass sowohl die Breite der rechtswissenschaftlichen Fächer berücksichtigt wurde, als auch die geografischen Unterschiede in Österreich. Man einigte sich darauf, das Team der Gründungseditors um ein Editorial Board zu ergänzen, in dem Mitglieder aus allen österreichischen rechtswissenschaftlichen Fakultäten als RepräsentantInnen der verschiedenen Fächer vertreten sind. So konnte der Anspruch, eine Plattform zu betreiben, die thematisch umfassende Forschung an österreichischem und europäischem Recht gleichermaßen verfolgt, erfüllt werden. Diese österreichweite Ausrichtung legitimierte den Titel „Austrian Law Journal". 5

Die Zielrichtung des ALJ ist für die österr rechtswissenschaftliche Publikationslandschaft aus mehreren Gründen innovativ: Zunächst durch die inhaltliche Universalausrichtung, die zwar den Fokus auf den nationalen Rechtsraum legt, jedoch ausdrücklich unionale, europäische und internationale Rechtsbereiche einbezieht.

$4 \mathrm{Vgl} \mathrm{dazu}$ de Vries/Roest/deJonge/Cuijpers/Munafò/Bastiaansen, The cumulative effect of reporting and citation biases on the apparent efficacy of treatments: the case of depression, Psychological Medicine 1-3 (2018), abrufbar unter <https://doi.org/10.1017/S0033291718001873> (15.9.2019).

5 Zu Beginn war noch ein „Grazer Law Journal“ angedacht: „Die Fakultät wird ein Konzept für die Herausgabe einer eigenen bzw. österreichweit herausgegebenen Fachzeitschrift erstellen, die online veröffentlicht werden soll (,Open Access') und einem wissenschaftlichen Begutachtungsprozess (peer review) unterliegt (,Grazer Law Journal')." (Zielvereinbarung zwischen Rektorat und der Rechtswissenschaftlichen Fakultät 2013 - 2015, 2). 
Zweitens, weil die wissenschaftlichen Hauptartikel ein double-blind peer review Verfahren durchlaufen und somit einem Begutachtungsprozess und einer Qualitätssicherung unterworfen sind. Drittens nutzt das ALJ als Open Access Zeitschrift aktiv die Möglichkeiten der IT und erlaubt damit die weltweite Dissemination von Forschungsergebnissen zu günstigsten Bedingungen. Schließlich kann die Einreichung nicht nur in deutscher, sondern auch in englischer Sprache erfolgen, um den globalen Austausch von Forschungsergebnissen zu fördern.

\section{Organisatorische Umsetzung}

In der Leistungsvereinbarung 2013-2015 verpflichtete sich die Universität Graz gegenüber dem Ministerium, im Rahmen einer Open Access Strategie an Kooperations- und Koordinationsaktivitäten der österreichischen Universitäten im Bereich von Open Access aktiv teilzunehmen und insb am Aufbau einer möglichst österreichweiten digitalen Publikationsplattform mitzuwirken. ${ }^{6}$ Dazu sollte bis 2015 ein frei zugängliches Repository eingerichtet werden, in dem die wissenschaftlichen Leistungen der Universität der breiten Öffentlichkeit zugänglich gemacht werden. ${ }^{7}$ Grundlage war das im Entwicklungsplan 2013-2018 definierte Bekenntnis der Universität zum Ausbau der „Digitalen Universität“. Der Verlag von Open Access Zeitschriften war damals jedoch noch nicht explizit angedacht. Das ALJ setzte insoweit einen Meilenstein, der insb durch enge Kooperation mit anderen Fakultäten $i Z m$ den technischen Grundlagen eines Open Access Journals möglich wurde.

\section{Technische Umsetzung}

Etwa zur gleichen Zeit, in der das Konzept des ALJ erarbeitet wurde, ermunterte der österr Fördergeber FWF, ${ }^{8}$ Projekte zu Open Access Journalen einzureichen. Dem folgten WissenschaftlerInnen der Universität Graz und reichten zwei erfolgversprechende Anträge ein. Die Aktivitäten führten zur probeweisen Einrichtung des Open Journal Systems (OJS) auf einem Server der UniIT.

OJS ist mandantenfähig und erlaubt so, verschiedene getrennte Angebote zu betreiben, die aus technischer Sicht (Wartung und Updates) zentral betreut werden können. Damit steht der Betrieb mehrerer, voneinander völlig unabhängiger Open Access Journale an der Universität Graz offen, deren technische Infrastruktur professionell an der UniIT verankert ist. Die Möglichkeit, in OJS eine eigenständige Homepage (Startseite) für jedes Journal zu administrieren, wurde vom ALJ aufgegriffen und ein Grafikbüro mit der Entwicklung von Logo und Sujets beauftragt.

6 Leistungsvereinbarung 2013 - 2015 zwischen der Universität Graz und dem Bundesministerium für Wissenschaft und Forschung, $28 \mathrm{f}$.

7 Dieses Repository wurde inzwischen als „uni-pub“ realisiert; Näheres dazu unter <https://ub.uni-graz. at/de/dienstleistungen/open-access/> (15.9.2019).

8 Details unter <https://www.fwf.ac.at/> (15.9.2019). 
Damit war im Frühjahr 2014 - obwohl die beiden Projektanträge in der zweiten Runde scheiterten - die technische Grundlage sowohl für das ALJ geschaffen, als auch für eine universitätsweite Infrastruktur, die bis heute sämtlichen MitarbeiterInnen und Institut(ion)en der Universität Graz zur Verfügung steht. ${ }^{9}$

\section{Zusammenarbeit mit der Universitätsbibliothek}

Von Anfang an wurde eine enge Anbindung an die Universitätsbibliothek gesucht, um bestehendes Know how und die universitätsweite Infrastruktur bestmöglich einzubinden. Damit ist die Langzeitarchivierung im Repository „uni-pub“ 10 ebenso gewährleistet wie die Nutzung der Suchmaschine des Universitätskatalogs (,,uni-kat" “) ${ }^{11}$ und bereits bestehender Indexierungen in DOAR, OAISTER, Primo Central Index etc. Die laufende Zusammenarbeit mit der Bibliothek startet nach Fertigstellung der PDF-Dokumente. Die gesamten verlegerischen und herausgeberischen Vorarbeiten verbleiben damit zur Gänze in der Verantwortung des ALJ.

\section{Finanzierung}

Das ALJ wurde im Wesentlichen von den Gründungsmitgliedern und ihren MitarbeiterInnen getragen. Dabei kam der Initiative zugute, dass in Person des Forschungsdekans und späteren Dekans Stefan Storr die Unterstützung durch einen wesentlichen Funktionsträger an der Fakultät gesichert war.

Die neben dem persönlichen Engagement aller Beteiligten - zu diesen zählen neben den Mitgliedern der Rechtswissenschaftlichen Fakultät gerade auch die MitarbeiterInnen von UniIT und Universitätsbibliothek - erforderlichen finanziellen Beiträge konnten in Form einer Anschubfinanzierung aufgebracht werden, die seitens des Vizerektors für Forschung, Univ.-Prof. Dr. Peter Scherrer, erfolgte. ${ }^{12}$ In seiner Person war dem ALJ ein weiterer Funktionsträger - nun auf Ebene des Rektorats von Anfang an positiv gesinnt.

\section{Betrieb $2014-2018$}

Das ALJ nahm seinen Betrieb mit der offiziellen Vorstellung am Fakultätstag der Rechtswissenschaften am 16. Mai 2014 auf. Damit waren Struktur und Rechtsrahmen festgelegt.

9 Währen eines der beiden Projekte völlig aufgegeben werden musste, konnte das zweite, das „Journal for Religion, Film and Media“ (<https://jrfm.eu>; [15.9.2019]) dank des persönlichen Einsatzes von ao. Univ.-Prof. Dr. Christian Wessely, Institut für Fundamentaltheologie an der Universität Graz, mit Anschubfinanzierung seitens des Vizerektors für Forschung letztlich doch realisiert werden.

10 <http://unipub.uni-graz.at> (15.9.2019); das ALJ ist dort abrufbar unter <http://unipub.uni-graz.at/ alj> (15.9.2019).

$11<$ <ttps://unikat.uni-graz.at> (15.9.2019).

12 Dabei wurde zur Vorbereitung im April 2014 und nach der offiziellen Präsentation am Fakultätstag im Mai 2014 die Grundausstattung des ALJ gesichert; ein weiterer Beitrag folgte im März 2015. 


\section{Struktur}

Das ALJ besteht aus den Editors, dem Editorial Board, dem Redaktionsteam und einem offenen Pool an GutachterInnen.

Der operative Kern der Zeitschrift liegt an der Universität Graz: HerausgeberInnen, MitarbeiterInnen, Serverstrukturen, technische Unterstützung sowie das Repositorium zur Langzeitarchivierung sind institutionell an der Karl-Franzens-Universität Graz verankert.

Die Editors tragen die Verantwortung für die Akquirierung der Beiträge, den peer review Prozess und die Veröffentlichung; sie handeln unentgeltlich. Die Herausgabe der Zeitschrift erfolgt gemeinsam mit dem Editorial Board, dessen Zusammensetzung gewährleistet, dass die großen Rechtsbereiche aller österr Rechtsfakultäten abgedeckt sind. Die Aufgabe der Mitglieder des Editorial Boards ist, Beiträge für das Journal anzuregen und bei AutorInnen einzuwerben, die Publikationswürdigkeit von Beiträgen vorab zu überprüfen und bei Bedarf geeignete GutachterInnen zu kontaktieren. Auch die Mitglieder des Editorial Boards handeln unentgeltlich.

Die GutachterInnen (Peers) handeln ebenso ohne Abgeltung; ${ }^{13}$ ihre Motivation entspringt der Verantwortung für die Förderung und Fortentwicklung der Rechtswissenschaften. Die Editors und die Mitglieder des Editorial Boards können dem Gutachterpool nicht angehören.

Die laufenden Redaktionstätigkeiten des ALJ umfassen neben der klassischen Betreuung der Texte auch die Kommunikationsaktivitäten des ALJ (Editors, Editorial Board, AutorInnen, Peers, InteressentInnen, LeserInnen, Verlage) sowie klassische Verlagstätigkeiten wie insb Satz, Lektorat und Bewerbung der Zeitschrift. Darüber hinaus liegt die Organisation von Veranstaltungen wie zB ALJ-Tagungen im Aufgabenbereich der Redaktion. Die Aufgaben werden derzeit mit einer 50\%-Stelle für wissenschaftliche MitarbeiterInnen gedeckt. Eine weitere Aufgabe ist die Unterstützung im englischsprachigen Lektorat, die seitens der Fakultät bereitgestellt wird.

Bezüglich zusätzlicher notwedniger Dienstleistungen im Bereich Technik und Archivierung kann auf das Know how und die Infrastruktur der Universität Graz, insb von UniIT und Universitätsbibliothek, zurückgegriffen werden.

\section{Rechtsrahmen}

Das ALJ ist eng an die Rechtswissenschaftliche Fakultät der Universität Graz angebunden. Da die Gründungseditors hier beschäftigt sind, wurde auch die redaktio-

13 Die anfängliche Praxis, den GutachterInnen einen Anerkennungsbetrag iHv € 100,- zukommen zu lassen, wurde bald eingestellt - weder hatten die Peers daran irgendein finanzielles Interessen, noch erwies sich der Verrechnungsaufwand als der Höhe des Betrags angemessen. 
nelle Tätigkeit in Graz verankert. Die Universität Graz tritt als Medieninhaberin des ALJ auf.

Inhaltlich bekennt sich das ALJ zu Open Access und stellt die veröffentlichten Artikel unter der Lizenz CC BY zur Verfügung. ${ }^{14}$ In der vorangehenden Diskussion wurde zwar thematisiert, ob eine kommerzielle Nutzung der Beiträge vorbehalten bleiben sollte (CC-BY-NC); die Entscheidung fiel aber in Anbetracht des vorrangigen Zieles breitestmöglicher Dissemination der Forschungsergebnisse letztlich dagegen aus. Diese offene Grundhaltung ermöglicht es, dass das ALJ in allen wichtigen kommerziellen Rechtsdatenbanken in Österreich abrufbar ist. ${ }^{15}$

\section{Organisatorische und institutionelle Verfestigung}

Da bei Gründung des ALJ Open Access Journale weder an der Fakultät noch an der Universität formell verankert waren, war die Adaptierung der universitären Lenkungsinstrumente ein wesentliches Desiderat, um die Nachhaltigkeit der Initiative sicherzustellen. Besondere Bedeutung kam dabei der förmlichen Anpassung des Entwicklungsplans im Jahr $2015 \mathrm{zu}$, in dem die Herausgabe des ALJ „als Online Zeitschrift (Open Access, peer reviewed) in Kooperation mit anderen rechtswissenschaftlichen Fakultäten in Österreich" ausdrücklich als Entwicklungsziel aufgenommen wurde. ${ }^{16}$ In der Folge verankerten die Universitäts- und die Fakultätsleitung ${ }^{17}$ das ALJ durch die Vereinbarung, „eine Redaktionsstelle“ für das ALJ finanzieren zu wollen. Tatsächlich wurde die Erfüllung der Aufgaben der Stelle auf mehrere Personen aufgeteilt, die an der Fakultät beschäftigt waren.

Inzwischen scheinen Open Access Journals an der Universität Graz fest verankert zu sein. ${ }^{18}$ In der Leistungsvereinbarung 2016-2018 wurde als Indikator für die Erreichung der Open Access Ziele der Universität Graz die Anzahl der Open Access Journale und Schriftenreihen definiert. ${ }^{19} \mathrm{Im}$ aktuellen Entwicklungsplan 2019-2024 genügt dazu ein generelles Bekenntnis, das Angebot der Universitätsbibliothek Graz an relevanten Metadaten und an elektronischen Volltexten im Bereich Open Access erweitern zu wollen, um die Verbreitung von Forschungsergeb-

14 Derzeit verwendet das ALJ CC BY 4.0 <https://creativecommons.org/licenses/by/4.0/deed.de> (15.9.2019).

15 Die Artikel des ALJ sind in LexisNexis, RDB, RIDA sowie der eLibrary (Verlag Österreich) im Volltext abrufbar.

16 Entwicklungsplan 2013-2018 Anpassung 2015, 36.

17 Zielvereinbarung zwischen dem Rektorat und der Rechtswissenschaftlichen Fakultät 2016 - 2018 , 3.

18 Mit Stand September 2019 werden 16 Open Access Journale und 4 Open Access Schriftenreihen an der Universität Graz betrieben; Details unter <http://unipub.uni-graz.at/obvugroaz> (15.9.2019). Weitere stehen in Planung.

19 Leistungsvereinbarung 2016 - 2018 zwischen der Universität Graz und dem Bundesministerium für Wissenschaft, Forschung und Wirtschaft, 33. 
nissen zu unterstützen. ${ }^{20} \mathrm{OA}$ Journals werden nicht mehr explizit erwähnt. In der Leistungsvereinbarung mit dem Ministerium hingegen wird wörtlich „[d]er Ausbau der Open Access Journale und Publikationen" neben dem offenen Zugang zu Literatur, Forschungsdaten sowie Lehr- und Lernmaterialien als „unentbehrliche Maßnahme“ bezeichnet, die durch die Zurverfügungstellung der erforderlichen digitalen Infrastruktur und elektronischer Workflows gewährleistet werden soll. ${ }^{21}$

\section{Publikationen}

Das ALJ bietet die Plattform, Beiträge aus allen Bereichen der Rechtswissenschaften zu veröffentlichen. Selbstverständlich besteht auch die Möglichkeit, die Ergebnisse rechtswissenschaftlicher Forschung, die auf Tagungen erzielt werden, nachhaltig zu dokumentieren.

Während frei eingereichte Beiträge („Hauptbeiträge“) jedenfalls den peer review Prozess durchlaufen müssen, wurden Tagungsbeiträge anfangs ohne Review-Verfahren angenommen. Im Jahr 2018 zeigte sich das ALJ aber ausreichend etabliert und nachgefragt, um alle Einreichungen an das Qualitätssicherungsverfahren zu binden.

Die Themen sind breit gestreut. Sie reichen von aktuellen Grundfragen der Rechtsphilosophie ${ }^{22}$ oder der bürgerlichen Identität der Frauen im Alten Rom, ${ }^{23}$ über neue Überlegungen zum „Beweglichen System“,24 bis hin zu kritischen Anregungen zum European Monetary Fund ${ }^{25}$ Negativzinsen ${ }^{26}$ oder der „Legalenteignung auf Grund von Symbolik“. 27

Insgesamt wurden bisher (Stand September 2019) 75 Beiträge veröffentlicht.

20 Entwicklungsplan 2019-2024, 16.

21 Leistungsvereinbarung 2019 - 2011 zwischen der Universität Graz und dem Bundesministerium für Bildung, Wissenschaft und Forschung, 7.

22 zB Somek, Authoritarian Liberalism, ALJ 1/2015, 67-87 (<http://alj.uni-graz.at/index.php/alj/article /view/37>) [15.9.2019]; Technokratie und Posttechnokratie, ALJ 2018, 58-65 (<http://alj.uni-graz.a t/index.php/alj/article/view/124> [15.9.2019]).

23 Peppe, Women and Civic Identity in Roman Antiquity, ALJ 1/2017, 23-38 (<http://alj.uni-graz.at/in dex.php/alj/article/view/71> [15.9.2019]).

24 Koziol, Das bewegliche System, ALJ 3/2017, 160-182 (<http://alj.uni-graz.at/index.php/alj/article/vi ew/111> [15.9.2019]).

25 Hofmeister, The Commission's Proposal to Establish a European Monetary Fund: A Critical Analysis, ALJ 2018, 139-158 (<http://alj.uni-graz.at/index.php/alj/article/view/126> [15.9.2019]).

26 Kronthaler, „Negativzinsen“ - Bestandsaufnahme und weitere offene Fragen, ALJ 2018, 26-57 (<http://alj.uni-graz.at/index.php/alj/article/view/121> [15.9.2019]).

27 Kirchmair, Das „Geburtshaus Hitlers“ in Braunau am Inn - eine unendliche Geschichte? ALJ 2018, 66-88 (<http://alj.uni-graz.at/index.php/alj/article/view/123> [15.9.2019]). 


\section{Organisation und nachhaltige Dokumentation von Veranstaltungen}

Neben der Publikation von Artikeln bildete die Kommunikation von Forschungsergebnissen in Präsenzveranstaltungen wie insb Konferenzen, Tagungen und Symposien, von Anfang an ein Desiderat, das im ALJ aktiv verwirklicht wurde.

So konnten zum Auftakt die Beiträge der Tagung „Recht, Governance und Verhaltensökonomik - eine interdisziplinäre Forschungsperspektive“ vom 28. April 2014 im ALJ publiziert werden. ${ }^{28}$ Ebenso wurden in Ausgabe 1/2015 die Ergebnisse einer Tagung des Instituts für Europarecht der Rechtswissenschaftlichen Fakultät der Universität Graz und des alumni UNI graz zu „20 Jahre Österreich in der Europäischen Union“ ${ }^{29}$ und in Ausgabe 1/2016 Beiträge zum „Thementag Recht und IT - Compliance Management" ${ }^{30}$ im ALJ veröffentlicht. Aber auch Einzelbeiträge aus Veranstaltungen können im ALJ publiziert werden. So fand jüngst mit Riss $^{31}$ der Beitrag einer Tagung im Rahmen eines Projekts zur Modernisierung des $\mathrm{ABGB}^{32}$ Verbreitung über das ALJ.

Daneben organisiert das ALJ selbsttätig Veranstaltungen. So wurden am 6. April 2017 die Tagung „Die digitalisierte Person“ 33 und am 1./2. März 2018 die Konferenz „Nudging and Information 2018: Is There a New Approach of Regulating the Behaviour of Consumers and Businesses?" abgehalten.

\section{Nutzungsstatistik}

Die Nutzung des ALJ erfolgt aufgrund der vielfältigen Referenzierungen in anderen Datenbanken äußerst weitschichtig. So werden ALJ-Beiträge beispielsweise über das Directory of Open Access Journals ${ }^{34}$ weltweit verbreitet und sind über das UlrichsWEB ${ }^{35}$ mit Schwerpunkt im deutschsprachigen Raum und über SHER$\mathrm{PA}^{36}$ im Vereinigten Königreich abrufbar. Die Indexierung bei Scopus und Westlaw wird derzeit verfolgt. Bemerkenswert ist, dass das ALJ in den österr kommerziellen

28 Details zur Veranstaltung unter <https://oeffentliches-recht.uni-graz.at/de/neuigkeiten/detail/article/r echt-governance-und-verhaltensoekonomik/> (15.9.2019); die Veröffentlichung im ALJ erfolgte in der ersten Ausgabe unter <https://alj.uni-graz.at/index.php/alj/issue/view/1> (15.9.2019).

$29 \mathrm{Vgl}<$ https://alumni.uni-graz.at/de/veranstaltungen/detail/article/jus-club-20-jahre-oesterreich-in-dereuropaeischen-union-ein-rueck-und-ausblick-aus-der-sicht-von-w/> (15.9.2019); die Beiträge sind veröffentlicht unter <https://alj.uni-graz.at/index.php/alj/issue/view/2> (15.9.2019).

$30 \mathrm{Vgl}<\mathrm{https} / /$ rewi-grundlagen.uni-graz.at/de/forschen/it-recht-und-rechtsinformatik/tagungenveranst altungen/11122015-compliance-management/> (15.9.2019); die Beiträge sind im ALJ abrufbar unter <https://alj.uni-graz.at/index.php/alj/issue/view/4> (15.9.2019).

31 Riss, Modernisierung des ABGB - Sachenrecht - Eigentumsrecht, ALJ 2018, 114 - 138, (<http://alj. uni-graz.at/index.php/alj/article/view/127> [15.9.2019]).

32 Details dazu unter <https://abgb-modernisierung.uni-graz.at/> (15.9.2019).

33 Veröffentlicht in ALJ, Ausgabe 2/2017, abrufbar unter <https://alj.uni-graz.at/index.php/alj/issue/vie $\mathrm{w} / 7>$ [15.9.2019].

$34<$ <ttps://doaj.org> (15.9.2019).

35 <http://ulrichsweb.serialssolutions.com/> (15.9.2019).

36 <http://sherpa.ac.uk> (15.9.2019). 
Rechtsdatenbanken RIDA, RDB, LexisNexis und in der eLibrary des Verlag Österreich Aufnahme gefunden hat.

Entsprechend vielschichtig gestaltet sich die Auswertung der Nutzungen: Die RDB meldete von Februar 2016 bis August 2019 exakt 7.813 Zugriffe von 5.602 Usern auf 1.654 ALJ-Beiträge; aus RIDA wurden seit 2015 bis Ende April 2018 genau 484 ALJ-Downloads gezogen. Uni-pub verzeichnet für die Jahre 2017/2018 um Webcrawler bereinigt 33.100 ALJ-Zugriffe, die nach den am öftesten angesehenen Artikeln gelistet werden und so die Interessen der LeserInnen deutlich machen. Das OJS-Auswertungstool erlaubt die Feststellung der Herkunft/des Standortes der LeserInnen nach Ländern. Danach griffen im 1. und 2. Quartal 2019 Personen aus 72 Ländern auf das ALJ zu, nämlich gereiht nach Häufigkeit der 10 bedeutendsten Staaten - Österreich, Deutschland, Vereinigte Staaten, Vereinigtes Königreich, Südkorea, Schweiz, Kanada, Italien, Indien, Niederlande. Nachdem im 2. und 3. Quartal 2018 Deutschland die Liste der Länderzugriffe angeführt hatte, steht nun (wieder) Österreich an erster Stelle:

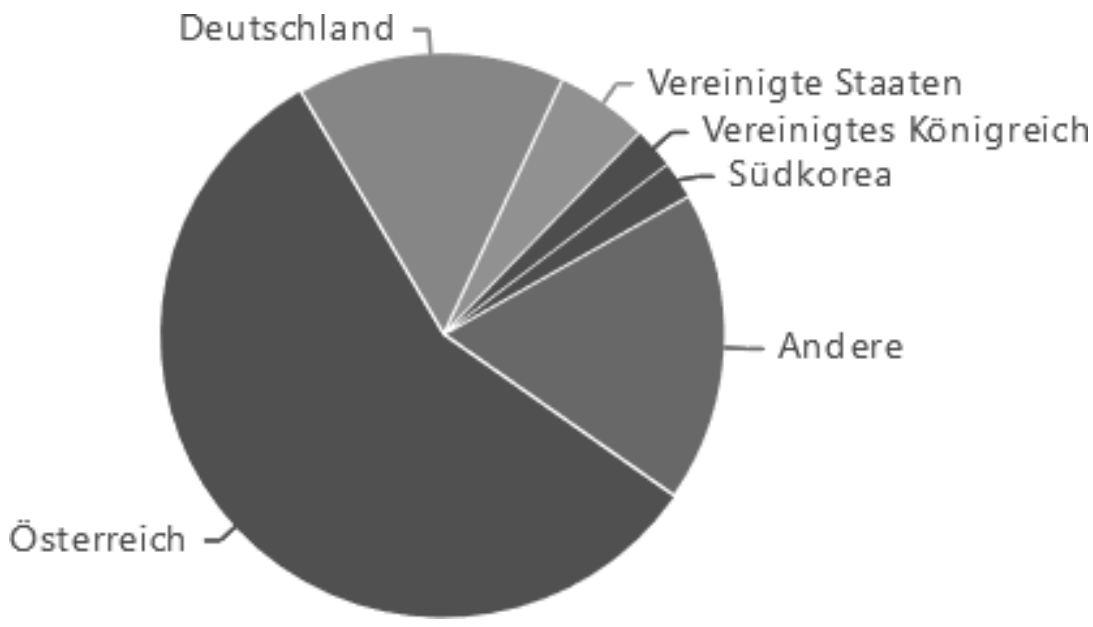

\section{Stand September 2019}

Der Wechsel von Stefan Storr an die Wirtschaftsuniversität Wien im Oktober 2018 brachte nicht nur der Fakultät einen neuen Dekan, sondern stellte, weil eine seiner Mitarbeiterinnen, die zentral die Redaktionstätigkeiten des ALJ betreut hatte, ebenfalls die Universität Graz verließ, das ALJ vor die Herausforderung einer wesentlichen Neuaufstellung.

Zunächst war abzuklären, ob das Team der ALJ-Editors auch überuniversitär besetzt sein kann. Diese Entscheidung, die die österreichweite Ausrichtung des ALJ auf die Probe stellte, wurde erfreulich schnell und einhellig bejahend getroffen. Als 
schwieriger erwies sich die Konsolidierung der redaktionellen Aufgaben, die auf vier Personen aufgeteilt waren. Während Redaktion und englischsprachiges Lektorat in Graz betreut wurden, wurde der Satz nach dem zwischenzeitigen Ausscheiden der Mitarbeiterin, die ihn durchgeführt hatte, extern vergeben. Sämtliche Aufgaben mit Ausnahme des englischsprachigen Lektorats sollen künftig durch eine $50 \%$ Stelle bewältigt werden.

Da keineswegs absehbar war (und ist), ob diese Bedeckung ausreicht, wurden die Aufgaben durch vorübergehende Aufstockung einer bestehenden Stelle am Institut für Rechtswissenschaftliche Grundlagen, Fachbereich Recht und IT, abgedeckt. Im 4. Quartal 2018 erfolgte durch bemerkenswertes Engagement der beteiligten Personen die Zusammenführung der Aufgaben auf eine Stelle und die Einschulung des neuen Mitarbeiters. Seit 1.1.2019 werden alle redaktionellen Aufgaben mit Ausnahme des englischsprachigen Lektorats von dieser Stelle im Ausmaß von 19 Wochenstunden bewältigt. Die Erfahrungen im Lauf des Jahres 2019 sollen zeigen, ob die Aufgaben des ALJ mit dieser Ausstattung erfüllt werden können bzw welches Stundenausmaß dafür erforderlich wäre.

Im Sommer 2019 wurde das ALJ einer Evaluierung seitens des Dekanats der rechtswissenschaftlichen Fakultät unterzogen. ...

Mit Stand September 2019 lässt sich zusammenfassend festhalten, dass sich das ALJ seit Mai 2014 zunehmend etabliert hat. Es ist heute eine anerkannte Plattform für die Publikation auch englischsprachiger hochwertiger, qualitätsgesicherter rechtswissenschaftlicher Texte, die häufig gerade in Projektanträgen eingefordert wird. ${ }^{37}$

\section{Best und Worst Practices sowie offene Themen}

\section{Best und Worst Practices}

Entstehung und bisheriger Betrieb des ALJ lassen die Feststellung von gelungenen und weniger gelungenen Teilschritten zu. Zu den best practices zählt, dass das ALJ von Anfang an die aktive Unterstützung universitärer Funktionsträger genoss, wie insb des (Forschungs-)Dekans und des Vizerektors für Forschung. Im gleichen Maß kann die bewusste Einbeziehung von Know how und Infrastruktur der Universität dazu gezählt werden, nämlich Universitätsbibliothek und IT-Abteilung. Als hilfreich und zweckmäßig erwies sich auch die (weitgehend formlose) Unterstützung seitens des Dekanats der Rechtswissenschaftlichen Fakultät. Das Engagement aller beteiligter Personen erwies sich aus organisatorischer Sicht letztlich als besonderer Glücksfall.

37 So erkennt insb der österr Fördergeber FWF die Veröffentlichung im ALJ als den Vorgaben „double blind peer reviewed" entsprechend an. 
Als Lehre bleibt die Erkenntnis, soweit möglich vorhandene universitäre Ressourcen zu nutzen und vorhandenes Know how anerkennungsvoll einzubinden.

Der Betrieb des ALJ machte recht bald die Anforderungen an die intensive Koordination und Kooperation der Beteiligten deutlich. Während die Sichtung und Bewertung der eingereichten Beiträge keinerlei Probleme darstellen und der Ablauf des double blind peer reviews den Beteiligten - Redaktion, Editors, Editorial Board und Peers sowie den AutorInnen - sehr rasch geläufig war, und selbst der Satz der Texte nach anfänglicher Auslagerung nun im Haus erfolgen kann, erwies sich der technische Ablauf in OJS als durchaus anspruchsvoll und gewöhnungsbedürftig. Immer wieder wurde der einfacheren Kommunikation per E-Mail der Vorrang gegeben. Diesbezüglich erwies sich die Konzentration der Redaktion im Herbst 2018 auf eine Person als wichtiger Schritt in die richtige Richtung. Das Anforderungsprofil umfasst sowohl fachliche Nähe (abgeschlossenes Diplomstudium Rechtswissenschaften) als auch Technikaffinität und Engagement im publizistischen Produktionsprozess.

Als verbleibender Schwachpunkt muss letztlich das Feld originärer Verlagstätigkeiten, wie unter anderem Marketing und Bewerbung außerhalb der klassisch-rechtswissenschaftlichen Modalitäten (wie insb Konferenzen und Tagungen) festgestellt werden, aber auch die grafische Gestaltung des ALJ. Letzterem konnte durch Beauftragung eines Grafikbüros zur Erstellung von Sujet und Logo begegnet werden; aktuell gestaltet eine Webdesignerin den Auftritt des ALJ von Grund auf neu. Die „Marktpräsenz“ eines kommerziellen Verlags konnte das ALJ hingegen bislang wohl nicht erringen.

Diesen Status teilt das ALJ mit anderen OJS-Zeitschriften der Universität Graz. Das führte Ende 2018 zu einer Petition an den Vizerektor für Forschung, in der angeregt wurde, den Universitätsverlag für diese unterstützende Funktion zu öffnen. Die HerausgeberInnen der AG-OJS an der Universität Graz bekennen sich darin nach wie vor zur Förderung der Wissenschaften durch den Betrieb von Open Access Journalen. Sie sind bereit, ohne erkennbaren finanziellen Ausgleich den Mehraufwand im Rahmen ihrer wissenschaftlichen Tätigkeiten für die Universität zu leisten. Aber sie ersuchen dringend, die Kompetenz des Universitätsverlags zu nutzen, um die Angebote professionell am Publikationsmarkt zu positionieren. Mit Unterstützung eines Verlages, der betriebswirtschaftlich agieren muss, dessen Ziel jedoch in erster Linie auf Kostendeckung, nicht jedoch auf Gewinnmaximierung gerichtet ist, könnte der Open Access Weg erfolgversprechend weitergeführt werden: Die HerausgeberInnen der Open Access Journale und Schriftenreihen könnten sich im Sinne ihrer Kernkompetenz auf die Erzeugung der Beiträge konzentrieren, während Bewerbung und „Vermarktung“ der Produkte über den Universitätsver- 
lag erfolgen würden. Die Entscheidung des Vizerektorats Forschung steht derzeit noch aus.

Als besonderer „Lerneffekt" aus dieser Phase soll festgehalten werden: Die Aufgaben der Beteiligten sollten ihren tatsächlichen Fähigkeiten entsprechen; WissenschaftlerInnen sind hervorragend in der Erzeugung wissenschaftlicher Beiträge; deren Vermarktung fällt ihnen aber oft deutlich schwerer. Diese Aufgabe überlasse man anderen, die sich damit auskennen.

In ganz engem thematischem Zusammenhang mit dem Gesagten steht die Einbeziehung des Editorial Board: Während Beiräte in Fachzeitschriften oft ein eher passives Dasein fristen, ist das Editorial Board des ALJ durch den peer review Prozess aktiv eingebunden. Nach einer ersten Sichtung der Beiträge durch die Editors wird das fachlich zuständige Board-Mitglied ersucht, mögliche Peers zu nennen und zu kontaktieren. Das nutzt nicht nur vorhandene Expertise, sondern fördert auch den Zusammenhalt im ALJ und soll damit als positives Beispiel zur Nachahmung empfohlen sein. Zugegebenermaßen könnte diese Aktivität aber auch im ALJ noch intensiver ausgebaut werden.

Weitere Erfahrungen, die noch nicht eindeutig als „best“ oder „worst“ practices zugeordnet werden sollen, betreffen die Zweckmäßigkeit des peer review Verfahrens, die Nutzung weiterer Möglichkeiten der IT wie zB Foren und Blogs und die Unentgeltlichkeit des Angebotes.

\section{Anonymität des peer review Prozesses}

Das peer review Verfahren wird inzwischen mehrfach kritisch hinterfragt. ${ }^{38}$ Aus Sicht des ALJ war anfangs vor allem die Anonymität als möglicherweise problematisch thematisiert. Die österr Landschaft rechtswissenschaftlicher FachautorInnen ist derart überschaubar, dass oft schon aus Thema und Stil erkennbar ist, wer den Text geschrieben hat. Dennoch hat sich daraus im Betrieb kein grundsätzliches Problem ergeben. Die Gutachten waren immer korrekt und sachlich. Die anfangs geäußerte Befürchtung, GutachterInnen könnten engstirnig oder gar eigennützig vorgehen, wurde in keinem einzigen Fall bestätigt. Allerdings hat sich bewährt, den Peers ein Formular an die Hand zu geben, nach welchen Kriterien sie sich bei der Begutachtung orientieren sollen. Das double blind peer review Verfahren kann und soll aus Sicht des ALJ jedenfalls als positive Erfahrung gewertet werden, die tatsächlich durch Anregungen die Verbesserung der Texte fördert. Wieweit es insgesamt dem Forschungsbetreib nachteilig sein könnte (im Sinne der eingangs er-

38 Dazu zählt zB, ob peer review vorgetäuscht wird (vgl dazu <https://www.economist.com/science-an d-technology/2018/06/23/some-science-journals-that-claim-to-peer-review-papers-do-not-do-so > [15.9.2019]) oder dass der Prozess die Forschungsergebnisse massiv verfälschen könnte (vgl dazu de Vries/Roest/deJonge/Cuijpers/Munafò/Bastiaansen, Psychological Medicine 1-3 [2018], abrufbar unter <https://doi.org/10.1017/S0033291718001873> [15.9.2019]). 
wähnten Bedenken in anderen Disziplinen) kann hier nicht ausgeführt werden, soll aber mahnend in Erinnerung bleiben.

\section{Nutzung weiterer IT-Möglichkeiten}

Das ALJ wurde als juristische Fachzeitschrift ausgerichtet, die inhaltlich ganz bewusst den Vorgaben traditioneller Angebote folgt. Die IT wurde ausschließlich dazu genutzt, die weltweite Verbreitung als Web-Angebot zu ermöglichen. Weitere Funktionen, die eine rege Diskussion von Forschungsergebnissen zulassen würden, wie zB Blog, Forum etc, wurden ganz bewusst hintangestellt. Ziel war es, das ALJ als seriöse, rechtswissenschaftliche Fachzeitschrift zu etablieren, ehe weitere Funktionalitäten angedacht werden. Im fünften Jahr seines Bestehens ist das ALJ dieser Ausrichtung treu geblieben; wir haben derzeit nicht vor, das Angebot auszuweiten. Dies vor allem auch, weil die Aufgaben bereits jetzt die zur Verfügung stehenden Ressourcen weitgehend binden. Hier stehen für die Zukunft aber durchaus Optionen offen.

\section{Unentgeltlichkeit des Angebots / Open Science}

Das ALJ hat sich, wie berichtet, ganz bewusst gegen jeglichen Vorbehalt kommerzieller Nutzung entschieden. Damit konnte zwar die Verbreitung der Beiträge durch die Aufnahme in die wesentlichen kommerziellen Rechtsdatenbanken in Österreich deutlich gesteigert werden, andererseits nimmt sich das ALJ damit jede eigenständige Einnahmequelle und bleibt auf die Finanzierung durch die Trägerorganisation (hier insb Universität Graz) angewiesen. Ob anstelle der CC-BY-Lizenz die kommerzielle Verwertung eingeschränkt werden sollte (CC-BY-NC), wäre breiter zu diskutieren.

In diesem Zusammenhang soll angemerkt sein, dass die Bemühungen der Europäischen Kommission um aktive Einbeziehung von Forschungsergebnissen als „Public Sector Information“, mit dem Ziel, sie zur gewerblichen wie nicht-gewerblichen Nutzung freizugeben, erfolgreich waren:

„Der Anwendungsbereich wird auch auf bestimmte Forschungsdaten - eine besondere Kategorie von Dokumenten, die als Teil der wissenschaftlichen Forschung erzengt wird - ausgeweitet, nämlich die Ergebnisse wissenschaftlicher Untersuchungen (Experimente, Erhebungen usw.), die die Grundlage des wissenschaftlichen Prozesses bilden; gleichzeitig bleiben Veröffentlichungen in wissenschaftlichen Fachzeitschriften weiterhin vom Anwendungsbereich ausgenommen, da sie zusätzliche Herausforderungen hinsichtlich der Rechtewahrnehmung mit sich bringen [...] Die kostenlose Bereitstellung von Dokumenten gilt insbesondere für For- 
schungsdaten und hochwertige Datensätze, die in einem delegierten Rechtsakt nach Artikel 13 festgelegt werden." 39

Der Vorschlag wurde nach Diskussion als RL (EU) 2019/102440 angenommen. Sie normiert die Verpflichtung der Mitgliedstaaten, „[...] Strategien für den offenen $\mathrm{Zu}$ gang in Bezug auf öffentlich finanzierte Forschungsdaten aufzustellen und dafür zu sorgen, dass diese Strategien von allen Forschungseinrichtungen und Forschungsförderungseinrichtungen umgesetzt werden $[\ldots]$ “ und zielt dabei insb auf sog „hochwertige Datensätze iSv Art 13 f RL (EU) 2019/1024. ${ }^{41} \mathrm{~J}$ edoch müssen Privatsphäre und personenbezogener Daten, die Geheimhaltung, die nationale Sicherheit, berechtigte Geschäftsinteressen, wie etwa Geschäftsgeheimnisse, und Rechte Dritter an geistigem Eigentum gemäß dem Grundsatz „so offen wie möglich, so geschlossen wie nötig" (as open as possible, as closed as necessary)" berücksichtigt werden. ${ }^{42}$ ErwGr 28 klärt dazu abschließend: „Andere Arten von Dokumenten als Forschungsdaten sollten weiterhin vom Anwendungsbereich dieser Richtlinie ausgenommen werden. " Die in aller Regel urheberrechtlich geschützten, wissenschaftlichen Texte - Monographien, Aufsätze, Artikel etc - bleiben also unberührt.

In eine ganz ähnliche Richtung weist die Strategie der Europäischen Kommission, sämtliche Wissenschaftsergebnisse in der „European Open Science Cloud“ (EOSC) zusammenzuführen und InteressentInnen zu öffnen. ${ }^{43}$ EOSC wurde Ende November 2018 in Wien gelauncht. Im Juni 2019 hat die EK einen Strategic Implementation Plan dazu veröffentlicht. ${ }^{44}$

Wie aber Universitäten sich am „Wissenschaftsmarkt“ bewähren sollen, wenn ihnen die Möglichkeit der ökonomischen Verwertung ihrer Leistungen genommen wird, wäre erst noch zu untersuchen und zu diskutieren. Es scheint, als habe sich Open Access in den letzten Jahren in erster Linie als Motor für gewerbliche und kommerzielle Tätigkeiten bewährt, ${ }^{45}$ während die Wissenschaft zunehmend Nachteile erkennt. Fake Journals, aber auch das double pricing renommierter Fachverla-

39 Vorschlag für eine Richtlinie des europäischen Parlaments und des Rates über die Weiterverwendung von Informationen des öffentlichen Sektors (Neufassung), KOM/2018/234 final, $11 \mathrm{f}$.

40 Richtlinie (EU) 2019/1024 des Europäischen Parlaments und des Rates vom 20. Juni 2019 über offene Daten und die Weiterverwendung von Informationen des öffentlichen Sektors, ABl L 2019/172, 56 (PSI-2-RL).

41 Die Task Force PSI und Open Data des BMDW ersucht - ausdrücklich „[a]ngesichts der besonderen Bedeutung der frühzeitigen Bewusstseinsbildung und Involvierung Österreichs in den Prozess der Ermittlung hochwertiger Datensätze“ - um Rückmeldungen und Feedback an psi@bmdw.gv.at.

42 Alle Originalzitate stammen aus Art 10 Abs 1 bzw dem diesen erläuternden ErwGr 28 PSI-2-RL (EU) 2019/1024.

43 Details dazu unter <https://ec.europa.eu/research/openscience/index.cfm?pg=open-science-cloud> (15.9.2019).

44 Abrufbar unter https://publications.europa.eu/en/publication-detail/-/publication/78ae5276ae8e-11e9-9d01-01aa75ed71a1/language-en.

45 Eine aktuelle, von MitarbeiterInnen der Universitätsbibliothek Graz durchgeführte Untersuchung in 82 kleinen und mittelständischen Wissenschaftsverlagen macht die Bedeutung von Open Access für 
ge zählen zu den bekannten Auswüchsen. Die erwähnte Studie zur Haltung der Verlage gegenüber Open Access hebt offene Finanzierungsfragen und mangelnde Anerkennung von Verlagsleistungen als wesentliche Probleme hervor und stellt den Wunsch der Verlage nach Standardisierung, Klarheit, administrative Vereinfachungen und verstärkte Kooperation mit Universitäten, Bibliotheken und Fördergebern fest. Open Access wird durchaus als "Zwangsbeglückung durch Fördergeber" verstanden. ${ }^{46}$ Letzteres erklärt sich aus der Ankündigung von Fördergebern, nur mehr Open Access publizierte Literatur finanzieren zu wollen (sog „Plan S“). ${ }^{47}$ Die StudienautorInnen prognostizieren, „[...] dass Verlage, die sich nicht an veränderte Anforderungen anpassen (wollen), nicht dauerhaft bestehen werden können."48 Das Bekenntnis ${ }^{49}$ des FWF zu „Plan S“ hat dennoch heftige Reaktionen in den Wissenschaften hervorgerufen. ${ }^{50}$

\section{E. Fazit}

Das ALJ kann im fünften Jahr seines Bestehens eine durchaus positive Bilanz ziehen. Es hat sich als seriöse, rechtswissenschaftliche Fachzeitschrift bewährt und nimmt einen fixen Platz in der österr Fachliteratur ein. Die Konzentration der redaktionellen Tätigkeiten in Graz bei gleichzeitiger Aufspaltung der Editors auf (derzeit) zwei österr Universitäten kann nach anfänglichen Unsicherheiten als ebenso gelungen bezeichnet werden, wie die Konzentration redaktioneller Tätigkeiten auf eine fachlich geeignete, technikaffine Person. Die Mitglieder des Editorial Board ergänzen die Expertise in fachlicher und räumlicher Hinsicht ebenso wie der ständig größer werdende Pool an Peers, die bereit sind, an der Verbesserung der eingereichten Texte aktiv mitzuwirken.

Ob Open Access in Form allgemeiner unentgeltlicher Zurverfügungstellung wissenschaftlicher Forschungsergebnisse insgesamt der richtige Weg in einer am „Wissenschaftsmarkt" orientierten Forschungslandschaft ist, wird aber noch ernsthaft zu diskutieren sein.

die Verlage deutlich: Kaier/Lackner, Open Access aus der Sicht von Verlagen, abrufbar unter $<$ https://edoc.hu-berlin.de/handle/18452/20410> (15.9.2019).

46 Kaier/Lackner, Open Access, 8, 14.

47 Details zu „Plan S“ unter <https://www.coalition-s.org/> (15.9.2019).

48 Kaier/Lackner, Open Access, 16.

49 <https://www.fwf.ac.at/en/news-and-media-relations/news/detail/nid/20181127-2339/?tx_rsmnews_ detail $\% 5$ Bref\%5D=1\&cHash=2cfbc919945edcf6073c55e3c2ed6544> (15.9.2019); weitere Informationen zu „Plan S“ unter <https://www.economist.com/open-future/2018/09/07/an-explosio n-of-openness-is-about-to-hit-scientific-publishing > und <https://www.deutschlandfunk.de/plan-s-fu er-open-access-bewegung-europaeische-foerderer.676.de.html?dram:article_id=427365> (15.9.2019).

$50<$ <https://science.orf.at/stories/2947392/> (15.9.2019). 